\title{
Présentation d'une méthodologie pour la prise de décision. Application à la reconnaissance automatique de signature acoustique
}

\author{
J.F. TROUILHET, I. COUSTAUX et J.P. GUILHOT
}

Laboratoire d'Acoustique de Métrologie et d'Instrumentation, 38 rue des 36 Ponts, 31400 Toulouse, France

\begin{abstract}
Résumé : Nous présentons une méthodologie de travail pour la résolution de problèmes de reconnaissance automatique de signature acoustique. Au delà des problèmes spécifiques à la reconnaissance d'un signal particulier, la majeure partie de la démarche est identique d'une signature à l'autre. En effet, l'étape d'analyse, l'essai de diverses méthodes de modélisation ainsi que le choix d'une méthode de classification sont les mêmes. Pour la résolution du problème de reconnaissance, nous décrivons les solutions envisageables pour chaque étape.
\end{abstract}

\begin{abstract}
This paper present a working methodology to solve the problem of automatic acoustic signature recognition. Ahead of the specifics problems due to a particular signal, for the most part, the procedures are identical from one signature to another. Indeed, the analysis step, the test of serveral modeling techniques and the choise of a classification method are the same.

We describe the conceivable solutions for each step for the resolution of a signal recognition problem.
\end{abstract}

\section{1 - IN T R O D U C T ION}

Le but de cette communication est de présenter une démarche applicable pour tout problème de reconnaissance automatique de signature acoustique. Nous allons voir que la majeure partie des opérations n'est pas spécifique au signal à reconnâ̂tre :

La reconnaissance d'un signal implique avant tout de bonnes connaissances sur celui-ci. Pour cela l'étape d'analyse du signal est très importante car elle peut permettre la mise en évidence de singularités propres au signal qui faciliteront d'autant plus sa reconnaissance.

En fonction de ces informations, le choix d'une méthode de modélisation devient possible. Celle-ci va fournir un ensemble de valeurs représentant le signal. De cet ensemble, on ne doit conserver uniquement qu'un sous ensemble afin de réaliser la classification. Il n'y a pas de technique ni pour le choix systématique de la méthode de modélisation ni pour le choix du sous ensemble des valeurs. Il devient alors important de juger de l'influence des choix effectués sur le résultat de la classification obtenue. En effet les résultats optimaux permettront de retenir les techniques à utiliser.

\section{2 - PRÉSENTATION DU SIGNAL À RECONNA ÎTRE}

Bien que cette présentation veuille couvrir un large domaine d'applications, l'illustration des méthođes exposées ici nécessite la mise en ouvre d'un cas concret. Pour cela, l'étude présentée consiste au comptage en temps réel du nombre de pontes de l'alose (Alosa alosa L. : un poisson migrateur de la Garonne) par reconnaissance du bruit caractéristique cré́ lors de la fraie [1]. Les difficultés pour la reconnaissance sont liées aux caractéristiques de ce signal. En effet celui-ci apparaît dans un environnement bruité, il est non stationnaire et n'est pas parfaitement reproductible dans le temps. Car ce n'est jamais le même couple de poissons qui produit le son, il n'y a donc aucune raison pour que ceux-ci soient identiques d'une ponte à l'autre. Les bruits sont dus, en majeure partie, aux véhicules routiers, aux trains, et enfin, aux divers animaux nocturnes : oiseaux, grillons, grenouilles ...

Le principal intérêt de cette étude est de permettre de connaître la fréquentation annuelle de chaque frayère et pour cela, il n'existe que la possibilité de compter sur place (ou en différé à partir d'enregistrements sonores) le nombre de pontes, caractérisées par un son particulier produit par la nage des poissons venus momentanément en surface. Près d'une quinzaine de sites doivent ainsi être étudiés chaque nuit de la saison 
migratoire (mai à juillet). Actuellement, pendant les phases de fraie (de minuit jusqu'au lever du jour), un magnétoscope fonctionne sur les lieux de ponte. Ensuite, par écoute de l'enregistrement, un chercheur comptabilise les bruits pour estimer la fréquentation des frayères moyennant la correction des pertes d'information liées à l'enregistrement acoustique. L'enregistrement est assuré par un microphone directement relié à un magnétoscope HI-FI qui présente une autonomie importante du fait de la programmation des créneaux d'enregistrement.

L'objet de cette étude est la réalisation d'un dispositif permettant la reconnaissance automatique du son produit par la fraie de l'alose en vue du dépouillement des enregistrements magnétiques en temps réel (voire accéléré) sans nécessiter l'intervention de l'utilisateur.

\section{3- MÉTHOD OLOGIE POUR LA RECONNAISSANCE}

\subsection{Méthodede test des résultats}

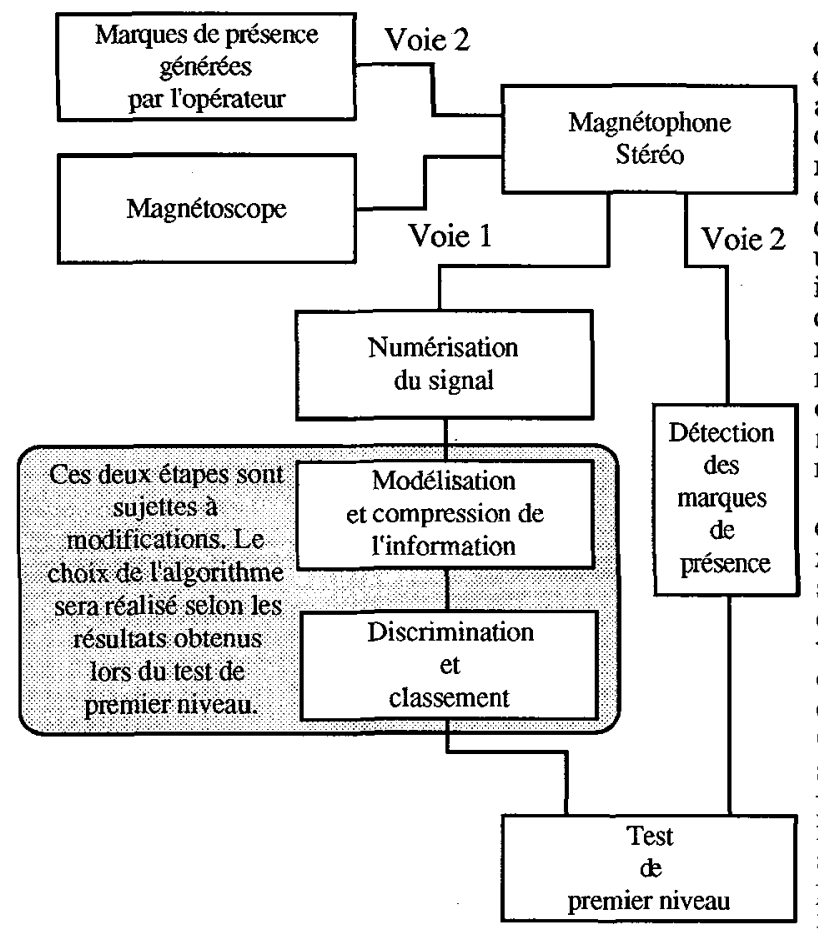

Les performances des diverses solutions doivent être évaluées afin de faire le meilleur choix. Les bandes magnétiques seront recopiees avec l'adjonction de marques de présence, sur la deuxième voie, ainsi plusieurs méthodes de reconnaissance pourront être évaluées entre elles et ceci de façon très systématique sans nécessiter de manipulation de la part de l'opérateur. Dans une première approche, on désire reconnaître les instants d'apparition du signal, et non le nombre de phénomènes. Pour cela, à l'aide d'une bande magnétique où l'apparition du signal est marquée par un expert, on compare les résultats obtenus à partir des méthodes imaginées avec la référence marquée sur la seconde voie du magnétophone (Fig. $\mathrm{n}^{\circ} 1$ ).

Figure $\mathrm{n}^{\circ} 1:$ Méthodologie de test des performances
Le marquage des bandes magnétiques par un expert ne peut être considéré comme une référence absolue. Én effet, en présence d'un signal non définissable mathématiquement, il est difficile de se prononcer sur sa présence dans tous les cas. La réérence, quoique parfaitement définie pour l'être humain, ne se prête pas à une comparaison dont le résultat doit être binaire. C'est le cas notamment au début et à la fin du son produit par l'alose ou encore lorsque le son parait très différent mais dont certains détails, impossibles à prendre en compte au niveau du système, permettent d'en attribuer l'origine au phénomène considéré. Pour ces raisons, les résultats des tests de performances des diverses méthodes sont à comparer entre eux plutôt qu'à une référence absolue.

L'univers des événements se divise en deux parties, une contenant les signaux où le son de l'alose est présent (A) et l'autre où il est absent. $(\overline{\mathrm{A}})$. Par contre le classificateur va créer deux zones légèrement différentes, une où il a déterminé la présence de l'alose $(\mathrm{dA})$ et l'autre où il ne l'a pas déterminée $(\mathrm{d} \overline{\mathrm{A}})$. Des quatre zones définies précédemment, on peut estimer les probabilités suivantes:

$$
\hat{\mathrm{P}}(\mathrm{dA} / \mathrm{A})=\frac{\hat{\mathrm{P}}(\mathrm{dA} \cap \mathrm{A})}{\hat{\mathrm{P}}(\mathrm{A})} \quad \begin{gathered}
\text { Probabilité de } \\
\text { détection }
\end{gathered} \quad \hat{\mathrm{P}}(\mathrm{dA} / \overline{\mathrm{A}})=\frac{\hat{\mathrm{P}}(\mathrm{dA} \cap \overline{\mathrm{A}})}{\hat{\mathrm{P}}(\overline{\mathrm{A}})} \quad \begin{gathered}
\text { Probabilité de fausse } \\
\text { alarme. }
\end{gathered}
$$

\section{2 - A nalyse du signal}

C'est la première etape et sûrement la plus importante. En effet, l'analyse du signal permet de caractériser et de mettre en évidence certaines particularités du signal. Par exemple, une périodicité cachée, la répartition de la puissance dans une certaine bande de fréquences, etc... Cette phase permet donc de fixer correctement les divers paramètres pour l'acquisition ultérieure du signal. De plus en fonction des singularités mises en évidence, elle permet de simplifier notablement les étapes suivantes. Dans le cas où le signal est stationnaire et en fonction de la durée d'observation, la transformation de Fourier ou encore une méthode de modélisation paramétrique permettraient l'analyse. Si celui-ci n'est pas stationnaire, on doit alors utiliser une transformation temps frequence (ex: Fourier glissant) ou encore une transformation temps-échelle (ex: Transformation en ondelettes). 


\section{3 - Modélisation du signal}

\subsection{1 - Type de modélisation.}

Pour obtenir de bonnes performances, il est important de réaliser la classification sur un faible nombre de paramètres dont le pouvoir discriminant a été reconnu. On peut discerner deux types de modélisations : Modélisation temporelle et modélisation fréquentielle du signal. Étant entendu que certaines modélisations permettent de revenir aux deux types de représentations. Mais il faut remarquer que de fortes variations dans le domaine temporel peuvent n'induire qu'une faible variation du spectre et vice-versa. Ainsi, selon que les paramètres représentatifs du signal seront plus proches d'une ou de l'autre des représentations, ils traduiront différemment cette variation. Par exemple si l'on considère une rampe de tension, une variation de la pente sera plus facilement discernable dans le domaine temporel que dans le domaine fréquentiel.

Une fois les caractéristiques du signal déterminées par l'étape d'analyse, nous pourrons choisir les conditions pour réaliser une acquisition et une modélisation correcte de celui-ci. Si le signal à reconnaître présente des singularités, nous orienterons la modélisation afin qu'elles soient représentées par les paramètres et ainsi faciliter la reconnaissance. A défaut, on peut utiliser une méthode de modélisation paramétrique qui permet d'obtenir un ensemble de parametres restreint.

\subsection{2 - Prise en compte des impératifs}

Hormis le coût, l'impératif le plus souvent rencontré est celui d'une classification en temps réel. Pour cela certains circuits intégrés permettant de mettre en neuvre différentes méthodes de classification. Toutefois la modélisation du signal devenant vite complexe, les processeurs de traitement numérique du signal trouvent ici une place privilégiée. La puissance de calcul de ces processeurs ayant des limites, il faut préférer les algorithmes récursifs a ceux mettant en cuvre des calculs complexes tels que l'inversion d'une matrice.

\section{4 - Classification}

\section{4 .1 - Pré-traitement des paramètres}

L'étape de pré-traitement des paramètres consiste à créer un nouveau vecteur paramètre à partir du vecteur initial issu de la modélisation. Selon l'information que l'on désire mettre en avant, on peut définir tel ou tel autre pré-traitement. Ces traitements peuvent être divisés en deux grandes familles selon que la classification se fera sur les vecteurs paramètres pris un à un ou qu'elle se fera avec prise en compte du temps. Selon le type de modélisation, on peut rediviser en deux chaque famille, approche non paramétrique ou paramétrique.

\section{4 .2 - Analyse des paramètres}

A partir du nombre important de paramètres que l'on peut obtenir lors de la phase de pré-traitement, on doit déterminer les plus pertinents pour réaliser la classification. Il est essentiel de noter qu'un nombre trop élevé de paramètres nuit à l'efficacité du classificateur. En effet, si nous prenons le cas d'un paramètre non pertinent, son effet sur le calcul des distances a pour effet d'ajouter une valeur aléatoire, donc d'introduire un flou supplémentaire. Le nombre de paramètres pour la classification doit être fonction du nombre et de la position des classes à séparer et non de la complexité du spectre du signal.

Grâce au critère de Fisher, nous pouvons évaluer la pertinence des paramètres pour un ensemble d'échantillons déjà classés par un expert. Le calcul du critère de Fisher nous permet de classer les paramètres par ordre de pertinence. Afin de réduire l'espace des caractéristiques, il ne restera plus qu'à réaliser une analyse factorielle discriminante. Les résultats obtenus par cette technique ne sont corrects que lorsque le nombre de classes indiquées par l'expert humain est égal ou supérieur au nombre réel de classes. Si cette condition n'est pas vérifiée, les résultats peuvent être complètement faussés car le critère est fonction de la valeur moyenne et de la variance des individus dans chaque classe. Ainsi dans le cas qui nous intéresse, nous désirons séparer les sons produits par la fraie de l'alose des autres sons. Mais ces autres sons regroupés dans une unique classe, par leurs différences entre paramètres, peuvent être mal représentés.

Pour être sûr que l'on n'est pas dans le cas décrit ci-dessus, il suffit de comparer les taux de détection, de la classification pour les paramètres pris un à un, et le critère de Fisher du paramètre correspondant. Si l'on note une corrélation pour l'ensemble des paramètres alors le critère de Fisher est significatif pour le regroupement effectué.

Les étapes de détermination des paramètres pertinents par le calcul du critère de Fisher et la réduction de l'espace des caractéristiques par une analyse factorielle discriminante, peuvent être remplacées par une analyse en composantes principales. Toutefois l'ACP est à déconseiller, car elle ne contribue qu'indirectement à l'amélioration du classement, puisqu'elle tend à maximiser la variance totale de l'ensemble des individus. Elle peut donc mettre en valeur un axe discriminant inutile pour la classification envisagée. Il vaut mieux utiliser l'AFD avec les indications de l'expert en tant que partition des classes.

De ce fait, dans certains cas, les résultats peuvent être totalement différents. Pour cela, nous préconisons un contrôle visuel de la répartition des paramètres et donc de leur pertinence. C'est un excellent moyen pour confirmer les résultats des méthodes précédentes qui sont plus systématiques. De plus, la méthode précédente est plus rapide que le cycle "classification et test des performances" qui n'indique pas le pourquoi des résultats obtenus. 


\subsection{3-Type de classification}

Les performances du classificateur réalisé dépendront non seulement de la méthode employée mais aussi des soins apportés lors de la réalisation de l'apprentissage. Si le nombre de classes à discerner est important, la classification de ceux-cí par un expert, en vue de l'apprentissage, devient vite fastidieuse voire impossible (Cf. [1] p:41-42). En effet, il ne faut pas, par exemple, classer tous les bruits crées par des véhicules dans une même classe. Il est plus sûr de laisser le système créer les partitions lui même, puis donner la possibilité à l'expert d'étiqueter (de donner un nom) ensuite chaque classe. Ces méthodes sont regroupées sous le nom de classification en mode non supervisé. Le but consiste à regrouper les $\mathrm{N}$ échantillons en nuages de points, selon que l'on commence avec une seule classe que l'on divise en plusieurs autres ou que l'on commence avec de nombreuses classes dont on réduit le nombre, on effectue respectivement un classification hierarchique descendante et une classification hiérarchique ascendante. Pour la classification, les deux méthodes les plus employées sont la méthode des K plus proches voisins KPPV et la classification par affectation au barycentre le plus proche qui découle de la théorie Bayésienne. La méthode de classification par affectation au barycentre le plus proche consiste a affecter le nouvel élément à la classe la plus proche de celui-ci au sens d'une distance donnée. La méthode des KPPV demande une place mémoire bien plus importante puisqu'elle nécessite de garder en mémoire tous les paramètres de tous les signaux. Elle a aussi une durée de calcul plus élevée puisqu'il faut calculer les distances du point à classer à tous les autres éléments. Cependant, elle donne très souvent en pratique de meilleurs résultats. En effet, la methode des distances Euclidiennes suppose des classes de forme sphérique dans un repère normé. Les hypothèses sur le signal à reconnâtre imposent des temps d'observation très longs, donc un nombre d'individus extrêmement élevé. C'est pour cela que nous avons retenu la méthode de classification par la distance au barycentre le plus proche qui est nettement plus rapide. De plus cette méthode pourra être réalisée, à terme, par un circuit spécialisé.

\section{4 - RÉSULTATS OBTENUS POUR LE SIGNAL À RECONNAÎTRE}

La solution retenue est exposée en détail dans la référence [2], en fonction des résultats obtenus lors du test des performances, nous avons adopté les solutions suivantes : Modélisation du signal : le spectre est estimé à partir d'une modélisation autorégressive obtenue à l'aide de l'algorithme de Levinson-Durbin. Nous avons choisi deux valeurs de la fréquence pour lesquelles la valeur du spectre permettra l'identification du signal. Classification : elle comporte deux phases : La phase d'apprentissage basée sur une méthode de classification non supervisée (Algorithme d'agrégation autour de centres mobiles) précédée d'une analyse factorielle discriminante. La phase de classification qui réalise la projection du modèle dans l'espace défini par l'analyse factorielle discriminante suivie du calcul des distances Euclidiennes aux diverses classes afin de déterminer l'appartenance du modèle à classer.

La prise en compte du temps est effectuée à l'aide d'une approximation polynomiale au sens des moindres carrés. Les meilleurs résultats ont été obtenus à partir d'une approximation polynomiale du premier ordre calculée à partir de vingt valeurs consécutives du spectre selon les équations suivantes :

$$
\begin{array}{ll}
\left.\hat{S}_{x}(t)\right|_{\hat{f}=0,1}=A_{1} \cdot t+A_{2} & \begin{array}{l}
\text { Évolution temporelle } \\
\text { du spectre pour les } \\
\text { fréquences f1 et } f 2
\end{array} \\
\left.\hat{S}_{x}(t)\right|_{\hat{f}=0,15}=A_{3} \cdot t+A_{4} &
\end{array}
$$

On obtient, avec une classification à partir des paramètres $\mathrm{A} 1$ et $\mathrm{A} 3$ les résultats suivants:

\begin{tabular}{|c|c|c|}
\hline $\mathrm{P}(\mathrm{dA} / \mathrm{A})$ & $\mathrm{P}(\mathrm{dA} / \overline{\mathrm{A}})$ & TRéussite \\
\hline 0,79 & 0,08 & $90 \%$ \\
\hline
\end{tabular}

Pour le problème présenté, un taux de fausse alarme de l'ordre de $10 \%$ est acceptable. Ainsi on obtient un taux de détection d'environ $79 \%$ et un taux de réussite de $90 \%$ pour la solution retenue.

\section{5 - CON CLUSION S}

Nous avons présenté une méthodologie appropriée à la résolution d'un problème de reconnaissance automatique de signature acoustique. Pour cela nous avons décomposé le problème en quatre phases d'égale importance : l'analyse, la modélisation, la classification et l'evaluation des performances atteintes. Ainsi nous avons pu retenir objectivement les solutions qui foumissaient le meilleur compromis probabilité de détection / probabilité de fausse alarme.

Nous avons utilisé avec succès cette méthode pour d'autres signaux de caractéristiques différentes. D'après les résultats obtenus, il semble que les performances de la classification soient largement fonction de la modélisation du signal et du pré-traitement des paramètres choisis. Nous présenterons ultérieurement les résultats de nos travaux actuels, l'évaluation d'une modélisation par ondelettes et d'un pré-traitement des paramètres par réseau neuro-mimétique.

\section{6 - B I B L I O G R A P H I E}

[1] J.F. TROUILHET : Étude et réalisation d'un dispositif de reconnaissance automatique de signature acoustique. Thèse de docteur de l'I.N.P. Toulouse - 11 juin 1992

[2] J.F. TROUILHET - I. COUSTAUX - J.P. GUILHOT : Reconnaissance des formes dans le plan tempsfréquence par modélisation paramétrique.

GRETSI Juan les pins. Septembre 1993, p:25-28. 